\title{
The spatial relation between EUV cavities and linear polarization signatures
}

\author{
Urszula Ba̧k-Stȩślicka ${ }^{1}$, Sarah E. Gibson ${ }^{2}$, Yuhong Fan ${ }^{2}$, \\ Christian Bethge ${ }^{2}$, Blake Forland ${ }^{3}$ and Laurel A. Rachmeler ${ }^{4}$ \\ ${ }^{1}$ Astronomical Institute, University of Wrocław, ul. Kopernika 11, 51-622 Wrocław, Poland \\ email: bak@astro.uni.wroc.pl \\ ${ }^{2}$ High Altitude Observatory, NCAR, P.O. Box 3000, Boulder, CO 80307, USA \\ email: (sgibson, yfan, bethge) @ucar . edu \\ ${ }^{3}$ Metropolitan State College of Denver, P.O. Box 173362, Denver, CO 80217-3362, USA \\ email: bforland@msudenver.edu \\ ${ }^{4}$ Royal Observatory of Belgium, Avenue Circulaire 3, 1180 Brussels, Belgium \\ email: rachmeler@oma.be
}

\begin{abstract}
Solar coronal cavities are regions of rarefied density and elliptical cross-section. The Coronal Multi-channel Polarimeter (CoMP) obtains daily full-Sun coronal observations in linear polarization, allowing a systematic analysis of the coronal magnetic field in polar-crown prominence cavities. These cavities commonly possess a characteristic "lagomorphic" signature in linear polarization that may be explained by a magnetic flux-rope model. We analyze the spatial relation between the EUV cavity and the CoMP linear polarization signature.
\end{abstract}

Keywords. Sun: corona; Sun: filaments, prominences; Sun: infrared; Sun: magnetic field

\section{Data Analysis and Results}

In our previous paper (Ba̧k-Stȩślicka et al. 2013) we have surveyed daily images from the Solar Dynamics Observatory/Atmospheric Imaging Assembly (SDO/AIA $193 \AA$, Lemen et al. 2012) for polar-crown cavities, and examined CoMP (Tomczyk et al. 2008) data to establish cavity signatures in linear polarization. Observations in linear polarization showed characteristic structures we termed lagomorphic, due to its resemblance to a rabbit-head seen in silhouette. We found 68 cases visible over the course of 78 days monitored. Using forward modeling we calculated synthetic CoMP-like data and we showed that lagomorphic structures are consistent with the flux rope model (e.g. Fan 2010).

In this paper we present an initial test of whether size of the cavity and size of the lagomorph are comparable, using a subset of cases including cavities with a good signal to noise in both AIA and CoMP and were large enough so that their center lay above the CoMP occulter. We compared the width of the EUV cavity and corresponding structures in the linear polarization. For this purpose we examined polar-angle cuts in SDO/AIA $193 \AA$ and CoMP L/I (degree of linear polarization) (see Figure 1 B-C) at the same height as the height of the center of the cavity seen on AIA $193 \AA$ images. We measured the average signal outside the cavity at the same height and defined a width using an area where the signal decreased more than $3 \sigma$ (black solid line on Figure 1 B-C). The relation between the width of the EUV cavity and the width of the lagomorph structures (for 16 cases) is presented on Figure 2. Analysis of the intensity profiles shows that the size of the lagomorph structure scales with the size of the cavity. 

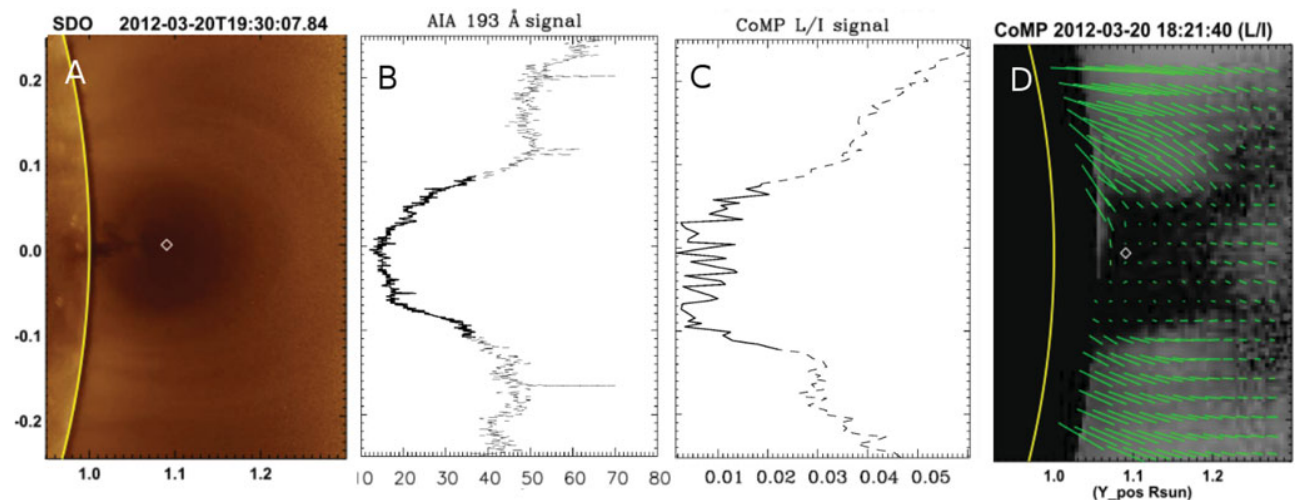

Figure 1. A) Example of cavity observed by SDO/AIA $193 \AA$ A. B) AIA $193 \AA$ intensity profile across polar-angle cuts at the height $1.09 \mathrm{R}_{\odot}$. Solid line shows $3 \sigma$ depletion. $\mathrm{C}$ ) L/I profile across polar-angle cuts at the height $1.09 \mathrm{R}_{\odot}$. Solid line shows $3 \sigma$ depletion. D) LOS-integrated L/I for CoMP observations. Direction of Stokes linear polarization vectors (integrated through the LOS) is shown as green lines. The edge of the solar disk is indicated by the curved yellow lines. White diamonds show the center of the cavity seen on AIA images.

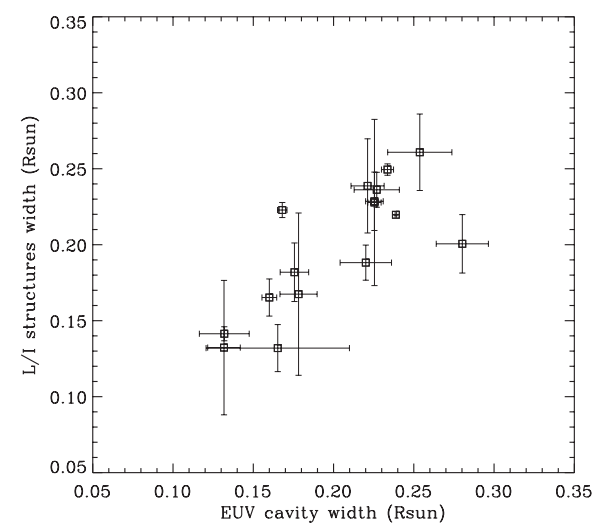

Figure 2. Relation between the width of the cavity seen on AIA $193 \AA$ images and the width of the lagomorph structure seen on CoMP L/I images (see text for details).

\section{Acknowledgements}

We thank Mark Miesch for an internal review of this manuscript. This work was supported in part by NASA LWS grant NNX09AJ89G to NCAR. UBS acknowledges financial support from the Polish National Science Centre grant 2011/03/B/ST9/00104. This work was enabled by participation of several of the authors in the International Space Science Institute (ISSI) working group on coronal magnetism. The CoMP data was provided courtesy of the MLSO, operated by the HAO, as part of the NCAR. NCAR is supported by the National Science Foundation.

\section{References}

Bạk-Stȩślicka, U., Gibson, S. E., Fan, Y., Bethge, C., Forland, B., \& Rachmeler, L. A. 2013, ApJ (Letters), 770, 28

Fan Y. 2010, ApJ, 719, 728

Lemen, J. R., Title, A. M., Akin, D. J., et al. 2012, Solar Phys., 275, 17

Tomczyk, S., Card, G. L., Darnell, T., Elmore, D. F., Lull, R. et al. 2008, Solar Phys., 247, 411 